\title{
UV-Written Bragg Gratings in a Flat-fiber Platform as a Bending and Twisting Sensor
}

\author{
S. Ambran, C. Holmes, J. C. Gates, A. S. Webb, J. K. Sahu, and P. G. R. Smith \\ Optoelectronics Research Centre, University of Southampton, Southampton, SO17 1BJ, United Kingdom
}

High demand on structural health monitoring has encouraged the development of smart structure geometries to be more effective and competitive [1]. One of the technologies that has been integrated into these structures is the fiber Bragg grating (FBG) [2]. The FBG is a mature technology that has seen many applications, particularly the field of sensing where it has many advantages such as immunity to electromagnetic field, long lifetime, high sensitivity, lightweight and low loss. There are several types of optically based physical sensors. Most of which are fabricated in a standard dimension silica optical fiber which due to its cylindrical structure are unable to independently measure twist. Here we demonstrate a bending and twist sensor fabricated in a flat-fiber substrate. A Y-splitter and a series of Bragg gratings along the $50 \mathrm{~mm}$ length provides a differential signal providing distinction between bend and twist within the sample.

The flat-fiber is a planar substrate made using standard MCVD (Modified Chemical Vapor Deposition) process [3]. Like conventional fiber it is flexible, low cost, low loss but with the added ability to support integrated circuits and thus also integrated sensing devices. The preform is collapsed on the fiber drawing tower with the help of a vacuum and produces the unique shape of the flat-fiber. The core layer is made photosensitive to UV by doping it with germanium and boron. This allows a UV writing technique to produce channel waveguides and splitters, as shown in Fig. 1(a). With the use of a two crossed-beams of the 244-nm argon ion laser, is possible to produce various period Bragg gratings simultaneously within the waveguides [4]. Several gratings with different periods may be defined within the device; this wavelength multiplexing provides a simple route to interrogate the local stress.

The device shown in Fig. 1(a) consists of a series of Bragg gratings at the input channel and both arms of the Y-splitter. One of the arms is placed near the center of the device and the other arm is located towards the edge of the flat-fiber. Monitoring multiplexed gratings in different spatial location allows discrimination of bending and twisting of the fiber.

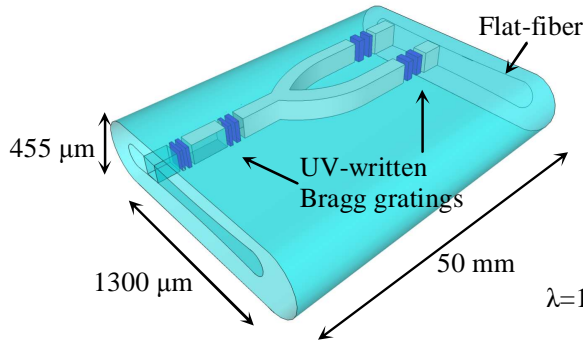

(a)

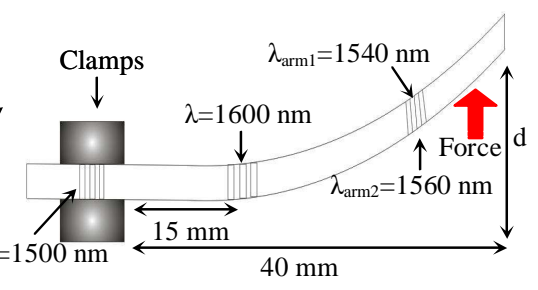

(b)

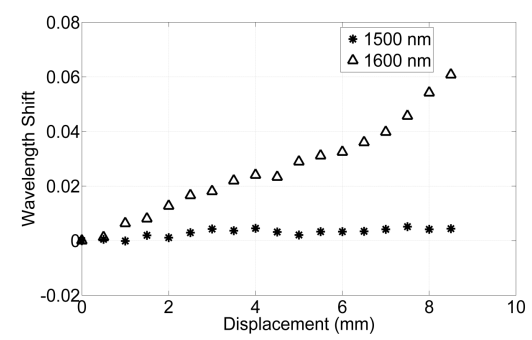

(c)

Fig. 1 (a) A 3-D diagram of UV-written Y-splitter waveguide in the flat-fiber platform; (b) A schematic of the bending experiment setup and (c) The Bragg grating wavelength change against the displacement, $d$ for $1500 \mathrm{~nm}$ and $1600 \mathrm{~nm}$ wavelength, the $1500 \mathrm{~nm}$ grating is a local temperature reference and does not respond to the stress.

In preliminary work the effect of bending a $50 \mathrm{~mm}$ long sample is shown. The measurement was taken by launching a light source into the input waveguide which was fixed at one point and the other end was displaced by a certain distance, $d$, as shown in Fig. 1(b). In this experiment two Bragg gratings are considered, the grating with wavelength of $1500 \mathrm{~nm}$ was located at the clamp point and was used as a temperature reference during the experiment. Whereas, the grating at $1600 \mathrm{~nm}$, located $15 \mathrm{~mm}$ away from the clamp point is used to measure the induced stress. Fig. 1(c) shows the Bragg wavelength shift of the two gratings as a function of displacement. The $1500 \mathrm{~nm}$ data shows a temperature variation of $\sim 0.5^{\circ} \mathrm{C}(\Delta \lambda \sim 5 \mathrm{pm})$ occurred during the experiment. The $1600 \mathrm{~nm}$ data exhibits a proportional relation between displacement and wavelength shift.

We shall present an optimized device design for the discrimination between twisting and bending stress.

\section{References}

[1] S. Liehr, P. Lenke, M. Wendt, K. Krebber, M. Seeger, E. Thiele, H. Metschies, B. Gebreselassie, and J. C. Munich, "Polymer optical fiber sensors for distributed strain measurement and application in structural health monitoring", IEEE Sens. Journal, 9, 11, (2009).

[2] A. Cusano, P. Capoluongo, S. Campopiano, A. Cutolo, M. Giordano, F. Felli, A. Paolozzi, and M. Caponero, "Experimental modal analysis of an aircraft model wing by embedded fiber Bragg grating sensors", IEEE Sens. Journal, 6, 1, (2006).

[3] A. S. Webb, F. R. Mahamd Adikan, J. K. Sahu, R. J. Standish, C. B. E. Gawith, J. C. Gates, P. G. R. Smith and D. N. Payne, "MCVD planar substrates for UV-written waveguide devices" Elec. Lett. 43, 9, (2007).

[4] G. D. Emmerson, S. P. Watts, C. B. E. Gawith, V. albanis, M. Ibsen, R. B. Williams, and P. G. R. Smith, "Fabrication of directly UVwritten channel waveguides with simultaneously defined integral Bragg gratings," Elec. Lett. 38, 1531-1532 (2002). 